\title{
MANAGEMENT OF DEMAND UNCERTAINTY WITHIN MRPII USING POSSIBILITY THEORY
}

\author{
${ }^{*}$ L.Geneste $^{1}$, B. Grabot ${ }^{1}$, G. Reynoso-Castillo ${ }^{2}$ \\ ${ }^{1}$ Equipe Production Automatisée - Laboratoire Génie de Production \\ Ecole Nationale d'Ingénieurs de Tarbes \\ 47, avenue Azereix, BP 1629 - F-65016 Tarbes cedex - France \\ ${ }^{2}$ Department of Applied Engineering, Panamerican University \\ Augusto Rodin \# 498 Col. Insurgentes Mixcoac \\ C.P. 03920. México D. F. - México
}

\begin{abstract}
In order to closely adapt what they produce to what will be sold, it is mandatory for nowadays companies to make early decisions on the base of an uncertain production planning. We suggest a way to explicitly model the uncertainty and imprecision of the demand using possibility theory, all along the MRPII steps: Material Requirement Planning, Load Planning, Scheduling. Though propagation of this uncertainty, the "Fuzzy-MRP" method aims at providing a richer information to decision makers, based on a quantification of the various situations that may arise according to the possible variations of the customer's demand. Copyright @2005 IFAC
\end{abstract}

Key words: Industrial production systems, planning, uncertainty, possibility theory, decision making

\section{INTRODUCTION}

Companies must nowadays constantly decrease their costs, and in that purpose closely adapt what they produce to the customer's demand. Since the demand only becomes certain on an horizon of time inferior to the product cycle time, it is necessary for that to make early decisions, on the base of an uncertain production planning. We suggest here to include in the model of the demand a subjective knowledge on its uncertainty, using the Possibility theory, and to adapt the main steps of the MRPII method in order to make possible to process this fuzzy information. Through this propagation of the uncertainty, the "Fuzzy-MRP" method aims at providing a richer information to decision makers, based on a quantification of the consequences of the possible variations of the customer's demand on the material requirements and on the load.

The paper is organized as follows: usual methods for dealing with uncertainty in a manufacturing system are presented in section 3 . The suggested framework for modeling an uncertain demand is described in section 4, whereas its possible use within the MRP method is shown in section 5 , with an emphasis on the load planning. Validation tests are described in section 6

\section{MANAGEMENT OF UNCERTAINTY IN MANUFACTURING}

Being able to cope with the uncertainty of the manufacturing environment is of course not a new concern. The MRPII (Manufacturing Resource Planning) method (Orlicky et al. 94) on which most of the production management pieces of software are based, uses three major ways for dealing with uncertainty: safety margins in the evaluation of quantities and delays, periodic refresh of the various production plans (i.e. rolling horizon plans) and safety stocks for being able to deal with an unexpected increase of the demand or with delivery problems. These techniques are generally achieved 
using probabilistic approaches. The idea is so to update periodically an expected demand implicitly considered as precise, which hardly allows the decision makers to have an early idea of the various situations which may arise. This could for instance be possible if the expected dispersion of the demand, known from the forecasts, was exploited. Many research works have been conducted in order to improve the way MRPII can handle the demand uncertainty, e.g. through improvements in the dimensioning of safety lead times (Whybark et al. 76), safety stocks (Grasso et al. 84), (Krupp 97), safety stocks and lot-sizing policy (Bodt et al. 83), (Ho et al. 98), (Brennan et al. 93), or by using only lot-sizing policies (Johansen 99). A state of the art on the dimensioning of safety stocks in a MRP context can for instance be found in (Guide et al. 00 ), concluding that no method clearly outperforms the others.

On the other hand, Fuzzy logic and Possibility theory have shown for a long time their interest for modeling information pervaded by imprecision and uncertainty, especially if this uncertainty is assessed on the base of a subjective appreciation. Most of the applications of these techniques to manufacturing concern scheduling (see for instance (Kerr et al. 89), (Fortemps 97), (Dubois et al. 89), (Dubois et al. 93)), but applications of fuzzy logic to higher planning levels can nevertheless be found, like (Inuiguchi et al. 94), where imprecise operation durations and preferences on sales at the level of the production plan are considered, or (Fargier et al. 00) where a representation of the imprecision on the ordered quantities is propagated in order to determine the global demand corresponding to a set of orders. However, all these approaches mainly deal with imprecision of data (ordered quantity for instance) and the possibility for an order to be cancelled is not addressed. In a recent work by the authors (Geneste et al. 03), an original scheduling technique has been proposed, based on an overbooking of resources, that enables to integrate uncertain orders. The method described hereafter aims at extending this approach to the whole MRPII algorithm. Suggestions related to material requirements and lot-sizing have previously been presented in (Reynoso et al. 02). This communication focuses on the load planning level and on the assessment of the interest of the method through simulation.

\section{POSSIBILISTIC MODELING OF AN ORDER AND BASIC OPERATIONS}

Our basic hypothesis, verified in many companies with which the authors have been in contact, is that a subjective knowledge on the uncertainty of the customers' orders exists, but is poorly exploited. The idea is here to use the possibility theory as a modelling framework in order to model the demand with all its uncertainty and imprecision, then to propagate it through the MRPII levels.

In that purpose, and on the base of a modeling framework originally described in (Dubois et al. 88), we suggest to represent the orders by a trapezoidal possibility distribution, as illustrated in Figure 1. The height of the trapezoid represents the possibility of occurrence of the order, ( 1 in the example of Figure 1), whereas its width represents the imprecision on the quantity. In Figure 1, we can for instance see that there is a non null possibility (here $h$ ') that the order is of 0 products, i.e. that the order is cancelled. If the cancellation of the order seems as plausible as its confirmation, h' will be equal to 1 . If the cancellation seems more plausible, the height of the trapezoid will be less than 1 whereas the value of $\mathrm{h}$ ' will remain equal to 1 . Within that framework, ignorance on the uncertainty of an order can be represented by $\mathrm{h}=\mathrm{h}^{\prime}=1$ (cancellation and confirmation are both plausible). Any kind of probabilistic representation requires further assumption, like supposing that the two eventualities have the same probability.

A trapezoid of height $h$ may be represented by 5 uples (a, b, c, d, h) as represented in Figure 1. (when $\mathrm{h}=1$, this notation will be simplified in $(\mathrm{a}, \mathrm{b}, \mathrm{c}, \mathrm{d})$ ). The order represented in Fig. 1 can consequently be described by:

$$
\pi(\mathrm{q})=\left(0,0,0,0, h^{\prime}\right) \cup(\mathrm{a}, \mathrm{b}, \mathrm{c}, \mathrm{d}, \mathrm{h})(1)
$$

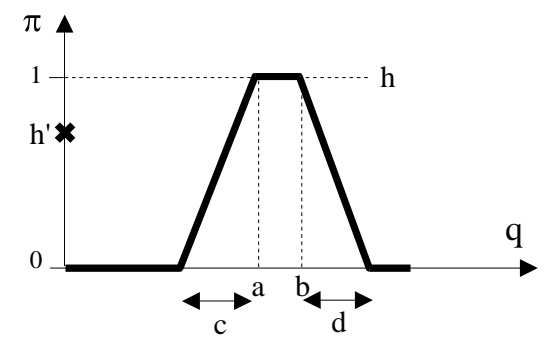

Fig. 1. Representation of an imprecise and uncertain order

As it will be shown later on, only addition, subtraction and multiplication by a scalar are required in order to process a fuzzy order through the basic steps of MRPII. These basic operations on trapezoidal fuzzy sets with a variable height are for instance defined in (Dubois et al. 88). Let us consider $\mathrm{A}_{i}=\left(\mathrm{a}_{i}, \mathrm{~b}_{i}, \mathrm{c}_{i}, \mathrm{~d}_{i}, \mathrm{~h}_{i}\right)$ and $\mathrm{A}_{j}=\left(\mathrm{a}_{j}, \mathrm{~b}_{j}, \mathrm{c}_{j}, \mathrm{~d}_{j}, \mathrm{~h}_{j}\right)$.

The sum and difference of $\mathrm{A}_{i}$ and $\mathrm{A}_{j}$ are defined as follows:

$$
\begin{array}{ll}
A_{i} \oplus A_{j}=(a, b, c, d, h) \text { with } & A_{i} \Theta A_{j}=(a, b, c, d, h) \text { with } \\
h=\min \left(h_{i}, h_{j}\right) & h=\min \left(h_{i}, h_{j}\right) \\
c=h \cdot\left(\frac{c_{i}}{h_{i}}+\frac{c_{j}}{h_{j}}\right) & c=h \cdot\left(\frac{c_{i}}{h_{i}}+\frac{d_{j}}{h_{j}}\right) \\
d=h \cdot\left(\frac{d_{i}}{h_{i}}+\frac{d_{j}}{h_{j}}\right) & d=h \cdot\left(\frac{d_{i}}{h_{i}}+\frac{c_{j}}{h_{j}}\right) \\
a=a_{i}+a_{j}-c_{i}-c_{j}+c & a=a_{i}-b_{j}-c_{i}-d_{j}+c \\
b=b_{i}+b_{j}+d_{i}+d_{j}-d & b=b_{i}-a_{j}+d_{i}+c_{j}-d
\end{array}
$$


We show in the next section how these operations allow to propagate imprecision and uncertainty within the MRPII steps, having for result the definition of the Fuzzy-MRP (F-MRP) method.

\section{F-MRP}

When a Master Production Planning is considered, the first step is to use the bills of materials for calculating which components are required. If a component $\mathrm{C}$ is used by products $\mathrm{A}$ and $\mathrm{B}$ during a period of time, the gross requirements on $\mathrm{C}$ will be the sum of the requirements on $\mathrm{A}$ and $\mathrm{B}$. Let us consider a set $\mathrm{O}$ of $\mathrm{n}$ orders of a given product $\mathrm{P}$ that should be provided at period $\mathrm{T}$.

$O=\left\{O^{1}, O^{2}, \ldots, O^{n}\right\}$, each order $O^{i}$ being described by a fuzzy union $O^{i}=A_{1}^{i} \cup A_{2}^{i} \ldots \cup A_{p_{i}}^{i}$ (see Fig. 1).

The gross requirements of $\mathrm{P}$ at period $\mathrm{T}$ are:

$\mathrm{GR}(\mathrm{P}, \mathrm{T})=\mathrm{O}^{1} \oplus \mathrm{O}^{2} \oplus \ldots \oplus \mathrm{O}^{\mathrm{n}}$

$=\left(A_{1}^{1} \cup A_{2}^{1} \ldots \cup A_{p_{1}}^{1}\right) \oplus\left(A_{1}^{2} \cup A_{2}^{2} \ldots \cup A_{p_{2}}^{2}\right) \oplus \ldots \oplus\left(A_{1}^{n} \cup A_{2}^{n} \ldots \cup A_{p_{n}}^{n}\right)$ $=\left(A_{1}^{1} \oplus A_{1}^{2} \ldots \oplus A_{1}^{n}\right) \cup\left(A_{1}^{1} \oplus A_{1}^{2} \ldots \oplus A_{2}^{n}\right) \cup \ldots \cup\left(A_{p_{1}}^{1} \oplus A_{p_{2}}^{2} \ldots \oplus A_{p_{n}}^{n}\right)$

Starting from the value of $\mathrm{GR}(\mathrm{P}, \mathrm{T})$ and taking into account the inventory level of product $\mathrm{P}$ at period $\mathrm{T}$, $\operatorname{Inv}(\mathrm{P}, \mathrm{T})$, the net requirements are equal to the gross requirements decreased by the level of inventory of the product, i.e.: $\mathrm{NR}(\mathrm{P}, \mathrm{T})=\mathrm{GR}(\mathrm{P}, \mathrm{T}) \Theta \operatorname{Inv}(\mathrm{P}, \mathrm{T})$

The next step of MRPII is to group the identified requirements according to a lot-sizing policy, e.g. lot-for-lot, sum of the demand on a given number of periods, fixed order quantity, etc. The first technique does not change the requirements, whereas the second one only requires to sum quantities, which is possible using the possibilistic addition previously described. The third case is more interesting, since it requires to satisfy a constraint on the size of each lot. In a classical calculation, a quantity of 40 parts required on period $n$, and 20 on period $n+1$ will for instance result in loted requirements LR of 50 parts on each period if the fixed quantity is 50: 30 parts are considered in excess on the second period in order to satisfy the constraint. In our case, this calculation can be done by computing the possibility and necessity degrees that the net requirements are more than each multiple of the lot size (see (Reynoso et al. 02)). The result of this process is a set of lots of the required quantity, each lot being associated with a possibility and a necessity degree, expressing to what extend the requirement on this lot is plausible.

We shall develop with more details the estimation of the workload generated by these requirements on each resource, which is the following step of MRPII. Let us consider as an example that two imprecise orders regarding different products $\mathrm{A}$ and $\mathrm{B}$ have to be considered, with $\mathrm{OA}=(5,10,2,5)$ parts and $\mathrm{OB}=(7,7,1,2)$ parts. Let us suppose that these products are manufactured on the same machine $\mathrm{M}$, with respective set-up times of $10 \mathrm{mns}$ for $\mathrm{A}$ and
$15 \mathrm{mns}$ for $\mathrm{B}$ and respective processing times per part of $20 \mathrm{mns}$ for $\mathrm{A}$ and $12 \mathrm{mns}$ for $\mathrm{B}$. We shall consider for simplification purpose that $\mathrm{A}$ and $\mathrm{B}$ are released according to a lot-for-lot policy and that no inventory is available for A nor B.

According to the previous formulae, the work load on $\mathrm{M}$, denoted WL(M), is:

$\mathrm{WL}(\mathrm{M})=($ set-upA $\oplus(\mathrm{LR}(\mathrm{A})$. processing_time $\mathrm{A})) \oplus$ (set-upB $\oplus(\mathrm{LR}(\mathrm{B})$. processing_timeB $)$ )

$=(10 \oplus((5,10,2,5)$. 20) $\oplus(15 \oplus(7,7,1,2)$. 12)

$=(10 \oplus(100,200,40,100) \oplus(15 \oplus(84,84,12,24)$

$=(110,210,40,100) \oplus(99,99,12,24)$

$=(210,309,52,124) \mathrm{mns}$

This step is illustrated in Figure 2 (central trapezoid of the left figure). It can be seen that the load on the resource on the period is known through a possibility distribution $\pi(\mathrm{w})$, which is a mode of representation rather far from the usual workload visualization in a load planning. Therefore, we suggest to derive from $\pi(\mathrm{w})$ the possibility that the real load is superior to $w$ for each $\mathrm{w}$ of the $\mathrm{x}$-axis.

Let $\mathrm{S}$ be the successive possible values of the load. We can calculate $\Pi(R \geq S)$ i.e. the possibility that the real load is higher than $S$, for $S$ varying from 0 to $\infty$ on the domain of the possible workload, using the formula:

$\Pi(R \geq S)=\sup (\pi(w))$ for $w \geq S$ (Dubois et al. 88).

It can be seen in Figure 2 that until $S$ is equal to the last value $\mathrm{w}$ for which $\pi(\mathrm{w})=1, \Pi(R \geq S)=1$. Then,

$\Pi(R \geq S)$ decreases according to the same slope than $\pi(\mathrm{w})$.

Only the right part of the possibility distribution has until now been exploited. In order to get a more comprehensive information, we can also calculate the necessity that the real load is superior to $w$ for each $\mathrm{w}$ of the $\mathrm{x}$-axis. In that case, we shall calculate $N(R \geq S)$ which is given by:

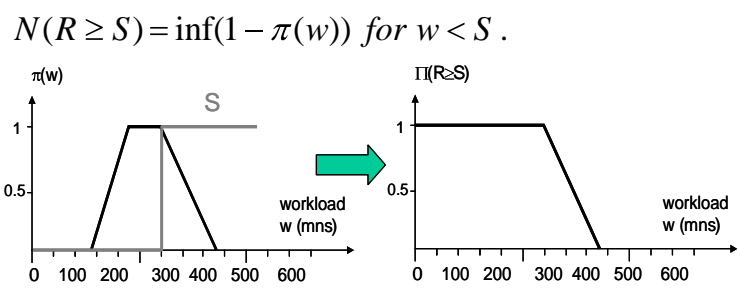

Fig. 2. Possibility that the load is superior to $\mathrm{w}$

The final result is summarized on Figure 3, on which it can be seen that, using a rotation of $90^{\circ}$, we obtain a visualization which is close to the bar graphs usually used for a load planning visualization, but which is much more informative. The darker area can be interpreted as the load necessarily present on the resource during the period of time, whereas the light gray area shows the load possibly present on the resource (the two areas are partially superposed in Figure 3, the possibility being greater than the necessity). 


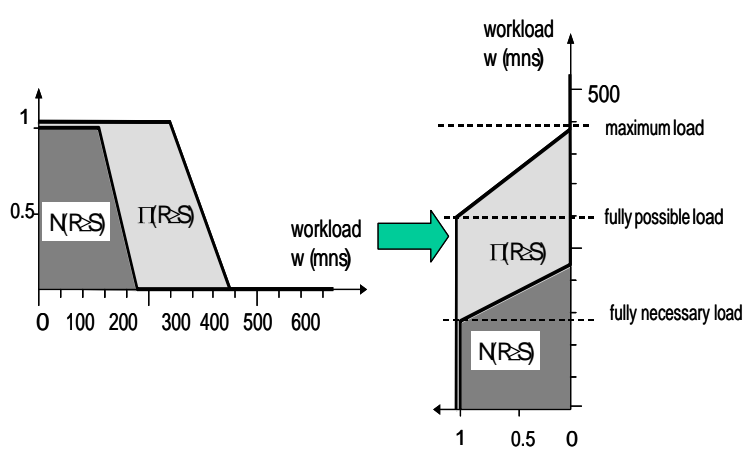

Fig. 3. Final visualization of the uncertain workload

It can be seen that the main interest of uncertainty propagation is to visualize at the same time a set of situations which may occur (here the various possible loads) together with a quantification of their possibility of occurrence (through the possibility and necessity degrees). It is therefore possible to prepare decisions for ensuring a good load / capacity balance, for example by warning subcontractors on the possibility of a later load transfer, by evaluating the feasibility of increasing the capacity of the workshop, or by load smoothing.

An example of load planning on several periods is shown in Figure 4. It can for instance be noted that the uncertainty is important on the first period, whereas the load on the fourth period is completely sure.

\section{VALIDATION TESTS}

In order to assess the interest of the F-MRP method, tests have been performed in order to compare it to a classical MRP method on various cases. In that purpose, the following parameters have been considered: numbers of resources, number of orders, number of products, structure of the bills of materials, level of uncertainty and imprecision of the demand. Five classes of imprecision and uncertainty have been defined for the orders, from crisp (0) to very imprecise (4), and from certain (0) to highly uncertain (4). Then, three levels have been defined for imprecision and uncertainty: in the first level of uncertainty, $60 \%$ of the orders are certain whereas $40 \%$ belong to class 1 (low uncertainty). In the third level, $60 \%$ of the orders belong to the class of highest uncertainty (4) and $40 \%$ to class 3 . Two parameters of the structure of the bills of materials have been considered: the number of levels (from 2 to 4) and the number of common components between articles.

After initial tests, it has been noted that the number of orders and resources have no influence on the results. A design of experiment has then been built using three levels of the remaining four parameters (numbers of levels of the bills of materials, component sharing, imprecision and uncertainty), leading to 27 experiments. These experiments have been conducted as follows:

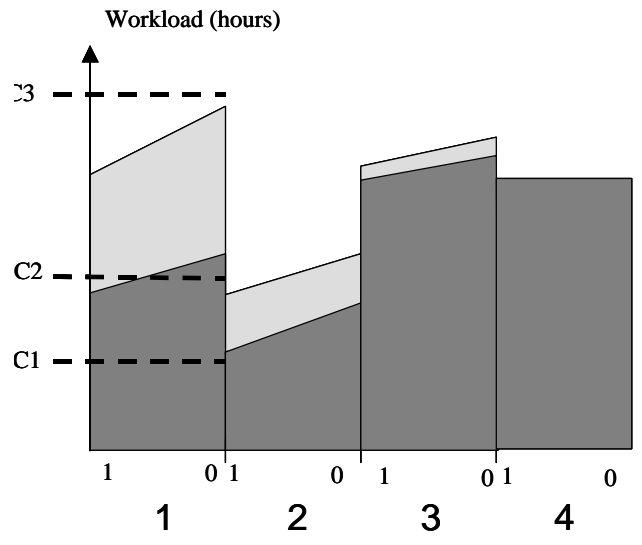

Fig. 4. Possibilistic load planning

- A list of orders is generated according to the values of the considered parameters.

- For MRP, the purpose has been to imitate as simply as possible the behaviour of a manager, who may have a more or less optimistic position regarding an uncertain demand. If he is very optimistic, he will plan all the orders, including the most uncertain ones. He therefore generates an excessive level of work in progress when some of the orders are cancelled. If he has a pessimistic behaviour, he will only plan certain orders, keeping the inventory level low but taking the risk of shortages. In order to model a full range of behaviours, we have applied successive levels of threshold on the uncertain demand, e.g. we denote MRP-0.9 the case when only orders with a possibility ( $h$ in Figure 1 ) superior to 0.9 are planned. Similarly, in case of an imprecise order, the manager has to choose the quantity of parts he will manufacture, between the minimum and the maximum plausible quantities. In the following figures, different choices have been represented, from planning the minimum value (right part of the figures) to the maximum value (left part). This way to integrate imprecise and uncertain orders allows to consider the full range of manager behaviour, and so to describe all the possible ways to use the method.

- For F-MRP, the orders are directly processed (uncertainty and imprecision are propagated). Nevertheless, the results have finally to be defuzzified when operational decisions must be made (e.g. for sending an order to a supplier). Like in previous case, different types of defuzzification are achieved, ranging from the minimum value (right part of the figures) to the maximum value (left part). As a consequence, the $\mathrm{x}$-axis showing the results (see Figures 5) is the same for MRP and FMRP (defuzzification from minimum to maximum) but it is important to keep in mind that it denotes an a priori defuzzification for MRP, and a defuzzification after imprecision and uncertainty propagation for F-MRP.

- We need then to assess the results of the two methods when confronted to a simulated real demand. For this, we have used an algorithm based on (Chanas et al. 88) which performs a possibilistic drawing, i.e. which confirms or cancels orders with 
respect to their initial possibility $h$, defined in Figure 2.

- The two methods are then compared according to the stock excess or shortage which occurs when the "real" (simulated) demand is compared to the planned one.

In Figure 5 is shown the average results of the 27 tests given by the design of experiment method. It can be seen that, for each combination of factors (uncertainty, imprecision, number of levels of the bill of materials, number of shared components), a defuzzification of F-MRP through the centre of mass (central curve) leads to rather good results (the curve cuts the $\mathrm{x}$-axis close to 0 , which means that no shortage nor over-inventory is generated). This is not the case for MRP, for which the upper curve (all the orders are considered) of course leads to overstocks, whereas the other strategies lead to more or less important shortages (bottom curves).

In order to assess the sensitivity of the results with regard to the parameters, only the best set-ups for MRP and F-MRP are now considered. Figure 6 shows the curves of the effects of the four factors for F-MRP, on the 27 tests. Let us remind that the effect of a factor at a given level (e.g. the effect of uncertainty at level 3) corresponds to the difference between the average of the results of the experiments in which the factor is set at the considered level and the average value of all the experiments.

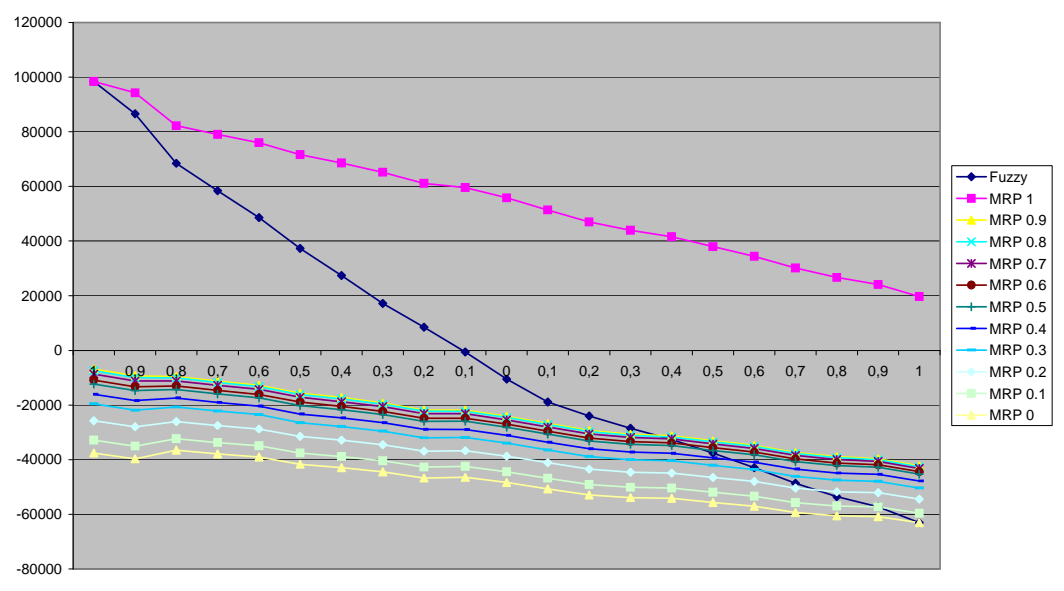

Fig. 5. Average results

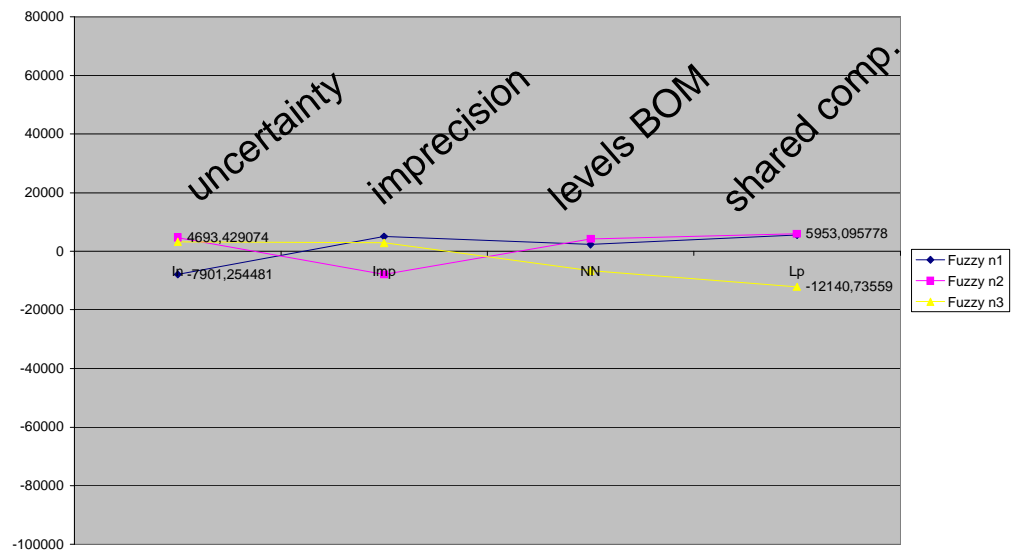

Fig. 6. Effects of the four factors for F-MRP
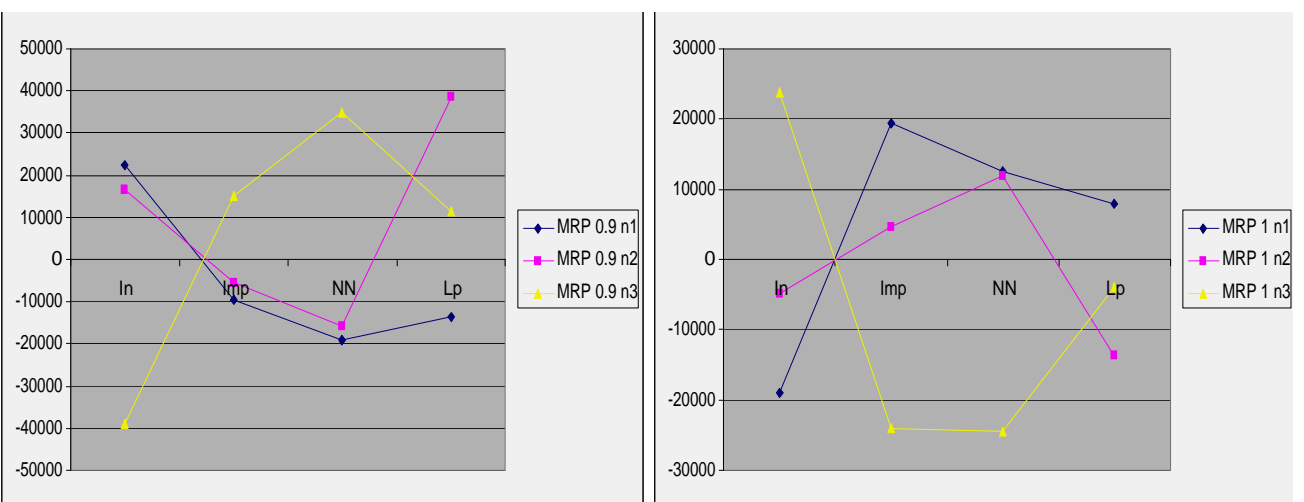

Fig. 7. Effects of the four factors for MRP 
In Figure 7 are shown the effects of the best set-ups for MRP (set-up of the threshold for considering the uncertain orders on the left graph, set-up of the quantity to consider on the right graph). It can be noticed that for these two set-ups, the effects are much more important than in Figure 6, especially for imprecision and uncertainty. These results show the interest of the suggested method for stabilising the effects on the results in situations where imprecision and uncertainty are important. Similar results have been obtained after tests regarding the level of overload or under-load in the loadplanning.

\section{CONCLUSION}

Using the suggested framework, a more rich information can be provided to the decision maker. Decisions requiring a long preparation (subcontracting, order of components, adjustments of capacity, etc.) can so be prepared earlier, on the base of quantified data, leading to better relations with the suppliers and to a better dimensioning of the internal inventories. Even if we showed the feasibility of an integrated management of uncertain orders in a MRP process, some problems remain as for its use in practice. Especially, an efficient use of the provided information requires to make it easily interpretable for a non-specialist of the possibility theory. Some answers have already been proposed (visualization of the production planning or of the workload for instance) but they can still be improved. In order to make a deeper validation of the method, a prototype software has been developed for allowing the processing of important examples within a more systematic validation approach. Using this prototype, tests are in progress in order to better assess the domain of interest of the suggested method regarding the percentage of uncertain orders, the level of uncertainty, and the level of imprecision of the considered demand.

\section{REFERENCES}

Bodt M.A., Van Wassenhove L.N. (1983) Cost increased due to demand uncertainty in MRP lot sizing, Decision Science Journal, 25, pp. 345362.

Brennan L., Gupta S.M. (1993) A structured analysis of material requirement planning under combined demand and supply uncertainty, International Journal of Production Research, 31 (7), pp. 1689-1707.

Chanas S., Nowakowski M. (1988) Single value simulation of fuzzy variable, Fuzzy Sets and Systems, 25 (1), pp. 43-57.

Dubois D., Prade H. (1988) Possibility theory, an approach to computerized processing of uncertainty, New York: Plenum.

Dubois D., Prade H. (1989) Processing Fuzzy
Temporal Knowledge, IEEE Transactions on Systems, Man and Cybernetics 19 (4), 729744.

Dubois D., Fargier, H., and Prade H. (1993) The use of fuzzy constraints in job-shop scheduling, IJCAI Workshop on Knowledge-Based Planning, Scheduling and Control, Chambéry (France).

Fargier H., Thierry C. (2000) The use of possibilistic decision theory in manufacturing planning and control: recent results in Fuzzy Master Production Scheduling in Scheduling under fuzziness, Ed. by R. Slowinski and M. Hapke, Springer-Verlag, 45-59.

Fortemps P. (1997) Jobshop scheduling with imprecise durations: a fuzzy approach, IEEE Trans. on Fuzzy Systems, 5 (4), 557-569.

Geneste L., Grabot B., Letouzey A. (2003) Scheduling uncertain orders in the customersubcontractor context, European Journal of Operational Research, vol.147, n², pp. 297 311.

Grasso E.T., Taylor B.W. (1984) A simulation based experimental of supply/timing uncertainty in MRP systems, International Journal of Production Research, 22, pp. 485-497.

Guide Jr V.D.R., Srivastava R. (2000) A review of techniques for buffering against uncertainty with MRP systems, Production Planning and Control, 11 (3), pp. 223-233.

Ho C.J., Ireland T.C. (1998) Correlating MRP system nervousness with forecast errors, International Journal of Production Research, 36 (8), pp. 2289-2299.

Inuiguchi M., Sakawa M., Kume Y. (1994) The usefulness of possibilistic programming in production planning problems, International Journal of Production Economics, 33, pp. 4552.

Johansen S.G. (1999) Lot sizing for varying degrees of demand uncertainty, International Journal of Production Economics, 59 (1-3), pp. 405-414.

Kerr R.M., Walker R.N. (1989) A Job Shop Scheduling System based on Fuzzy Arithmetic, $3^{\text {rd }}$ International Conference on Expert Systems and the Leading Edge in Production and Operation Management, South Carolina, USA, 433-450.

Krupp J.A.G., (1997) Safety stock management, Production and Inventory Journal, $3^{\text {rd }}$ qu., pp. 11-18.

Orlicky J., Plossl G. (1994) Orlicky's Material Requirement Planning, McGraw Hill Text, 2nd Edition.

Reynoso G., Grabot B., Geneste L., Vérot S. (2002) Integration of uncertain and imprecise orders in MRPII, 9th Int. Multi-Conf. on Advanced Computer Systems, Miedzyzdroje, Poland, October 23-25.

Whybark D.C., Williams J.G. (1976) Material requirements planning under uncertainty, Decision Science Journal, 7, pp. 595-606. 